\title{
Sternal lifting increases the operating space in esophagectomy via mediastinoscopy: a prospective cohort study
}

\author{
Shaojin Zhu ${ }^{1} \wedge$, Gengxin Zhang ${ }^{1}$, Qi You ${ }^{1}$, Fei Li $^{1}$, Boying Ding ${ }^{1}$, Feng Liu ${ }^{2}$, Tongjun $\mathrm{Ma}^{3} \wedge$ \\ ${ }^{1}$ Department of Thoracic Surgery, The First Affiliated Hospital of Wannan Medical College (Yijishan Hospital of Wannan Medical College), Wuhu, \\ China; ${ }^{2}$ Department of Thoracic Surgery, Lishui Branch of Zhongda Hospital Affiliated to Southeast University, Nanjing, China; ${ }^{3}$ School of Forensic \\ Medicine, Wannan Medical College, Wuhu, China \\ Contributions: (I) Conception and design: T Ma, S Zhu, F Liu; (II) Administrative support: T Ma, B Ding; (III) Provision of study materials or \\ patients: S Zhu, G Zhang, Q You; (IV) Collection and assembly of data: G Zhang, Q You, F Li; (V) Data analysis and interpretation: S Zhu, G \\ Zhang, Q You, F Li; (VI) Manuscript writing: All authors; (VII) Final approval of manuscript: All authors. \\ Correspondence to: Tongjun Ma. School of Forensic Medicine, Wannan Medical College, Wuhu 241002, China. Email: mtj@wnmc.edu.cn.
}

Background: Esophagectomy with combined single-port inflatable mediastinoscopy and laparoscopy reduces the risk of postoperative respiratory complications as it obviates the need to pass through the pleural space. However, it has strict indications owing to the narrow space for operation. Therefore, we adopted a sternal lifting method using a retractor that enables the expansion of the operating space, a technique which has not been previously reported. We describe our experience and report the results of an evaluation of this new approach.

Methods: Thirty-nine patients with esophageal squamous cell carcinomas underwent esophagectomy using combined single-port inflatable mediastinoscopy and laparoscopy from March 2019 to August 2021. Among them, 20 cases received sternal suspension [sternal suspension group (SS group)], and 19 cases did not receive sternal suspension [non-sternal suspension group (NSS group)]. The short-term efficacy of the two groups was observed.

Results: Patients in the SS group had a shorter intramediastinal operation time (82.50 vs. 110.00 minutes; $\mathrm{P}<0.001)$, more dissected chest lymph nodes (14 vs. 12; $\mathrm{P}=0.036$ ), and a lower incidence of postoperative hoarseness ( 2 vs. $6 ; \mathrm{P}=0.235)$ than did those in the NSS group. There were no significant differences between the SS group and NSS group in terms of intraoperative blood loss, postoperative hospital stay, post-surgical pathologic TNM classification (pTNM), post-surgical pathologic tumor classification (pT), post-surgical pathologic extent of lymph node involvement $(\mathrm{pN})$, and total number of dissected lymph nodes. There were no statistical differences in the incidence of anastomotic fistula, respiratory complications, arrhythmia, or chylothorax between the two groups. There was no mortality during hospitalization in the two groups.

Conclusions: Sternal lifting increases the working space in esophagectomy via mediastinoscopy. It can make video-assisted radical esophagectomy by a transmediastinal approach with total pneumomediastinum assistance (VARETT) easier to perform, and sternal suspension in VARETT is safe and effective.

Keywords: Esophagectomy; mediastinoscopy; sternal lifting; the operating space

Submitted Aug 19, 2021. Accepted for publication Sep 15, 2021.

doi: $10.21037 /$ jtd-21-1406

View this article at: https://dx.doi.org/10.21037/jtd-21-1406

^ ORCID: Shaojin Zhu, 0000-0002-0633-3987; Tongjun Ma, 0000-0001-8093-4900. 


\section{Introduction}

Esophageal cancer is a malignant tumor that seriously threatens the people's health. Of all treatments, surgery remains the most effective treatment for esophageal cancer $(1,2)$. Postoperative respiratory complications are the most common following open surgery esophagectomy (Sweet, Ivor Lewis, or McKeown esophagectomy) or thoracoscopy combined with laparoscopy. Recently, esophagectomy using combined single-port inflatable mediastinoscopy and laparoscopy, also called video-assisted radical esophagectomy by a transmediastinal approach with total pneumomediastinum assistance (VARETT), has gained interest since it was first reported by Fujiwara in 2015 (3). It is reported to effectively reduce the risk of postoperative respiratory complications because it does not pass through the pleural space while preserving the bronchial artery and the pulmonary plexus of the vagus nerve (4). Some studies have shown that VARETT further reduces surgical trauma and appears to be more beneficial in protecting respiratory function compared to combined thoracic laparoscopic surgery (5-7).

However, VARETT has strict indications because of the narrow space for mediastinoscopically separating the esophagus under insufflated carbon dioxide, resulting in a small operative field (8) and making the operation particularly challenging. Thus, this procedure is only generally suitable for patients with T1-2N0M0 cancers. It is only used as an alternative to routine surgery for patients with early esophageal cancer with cardiopulmonary dysfunction because of the limited space available for surgery. Therefore, VARETT cannot be widely applied (9).

To solve the problem of a small operating space, we developed a new surgical technique in which the sternum is suspended during surgery. This sternal lifting involves using a retractor to expand the operative field in VARETT, making it easier to separate the esophagus. Herein, we report our experience with this technique. The purpose of this study was to preliminarily explore the feasibility, safety, general efficacy, and short-term clinical efficacy of adding sternal suspension during VARETT. We present the following article in accordance with the STROBE reporting checklist (available at https://dx.doi.org/10.21037/jtd-211406).

\section{Methods}

Our study was designed as a prospective cohort observational study. The participants included were esophageal squamous cell carcinoma patients who were undergoing VARETT. Patients were divided into two cohorts based on whether sternal suspension was added during VARETT. Basic data, intraoperative and postoperative conditions, and routine follow-up were collected from admission. The surgeon had extensive experience in endoscopic esophagectomy, and had completed animal trials of this procedure before initiating clinical VARETT (8).

\section{Patients}

Thirty-nine patients with esophageal squamous cell carcinoma underwent esophageal VARETT surgery in the Thoracic Surgery Department of the First Affiliated Hospital of Wannan Medical College (Yijishan Hospital) from March 2019 to August 2021. After explaining the advantages and disadvantages of sternal suspension to the patient, the attending physician decided whether to use sternal suspension during the operation according to the patient's own choice. Twenty patients had a sternal suspension added during VARETT [sternal suspension group (SS group)] and 19 cases did not receive sternal suspension [non-sternal suspension group (NSS group)]. All patients were from the same hospital. Both groups were operated on by the same surgical team. The study was approved by the institutional ethics committee of Yijishan Hospital of Wannan Medical College (No.: 73). All patients undergoing surgery signed informed consent forms. The design of the study was carried out in strict accordance with the principles of the Declaration of Helsinki (2013 revision). There were no significant differences in age, sex, or other basic data between the two groups (Table 1).

\section{Inclusion criteria}

Patients in the study were required to meet the following criteria: (I) endoscopic biopsy histological diagnosis of squamous cell carcinoma, CT1-3N0-1M0; (II) no distant organ metastasis anywhere in the body; and (III) good function of all organs.

\section{Exclusion criteria}

Patients with one of the following symptoms were excluded: (I) a previous history of esophagectomy, gastrectomy, or mediastinal surgery; (II) diseases currently not under control, such as severe heart disease, uncontrolled 
Table 1 Comparison of baseline demographics between the two groups

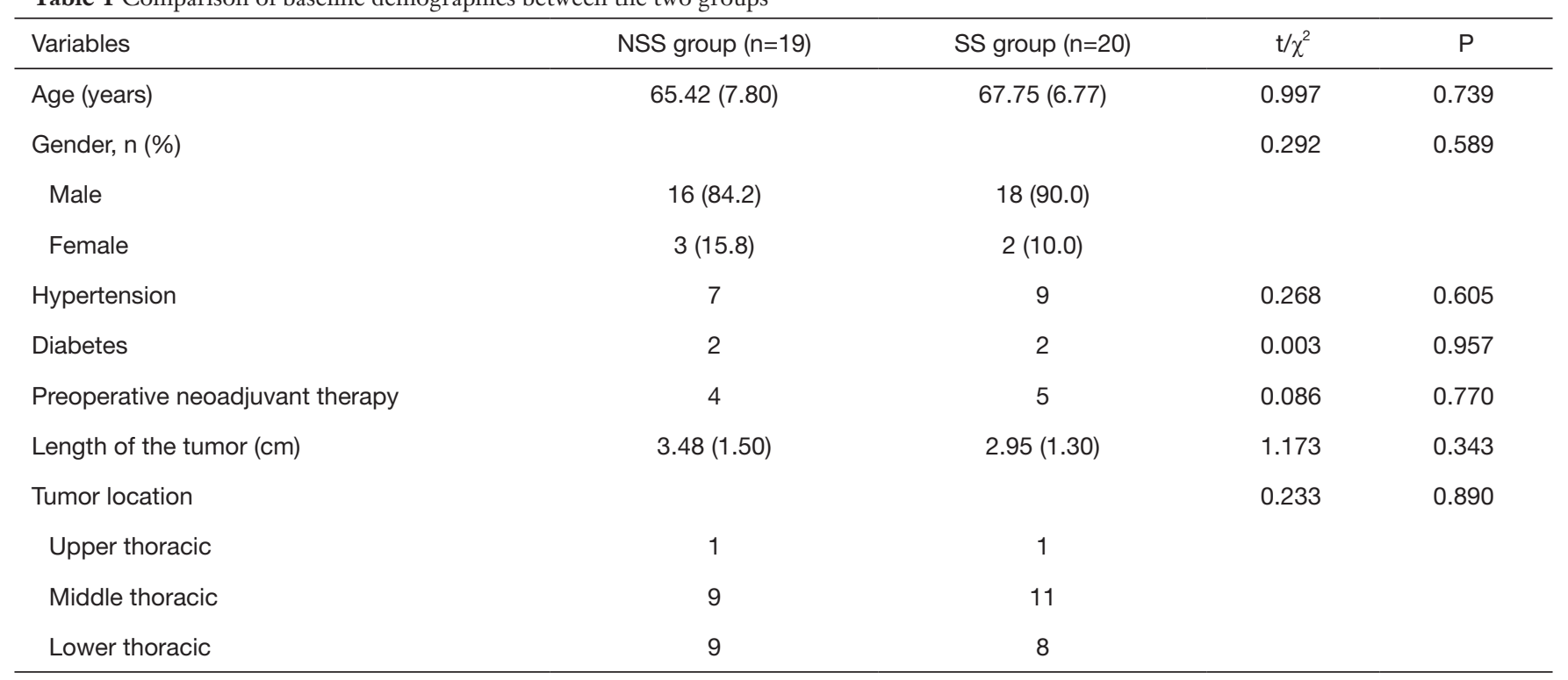

NSS group, non-sternal suspension group; SS group, sternal suspension group.

hypertension or diabetes, active bacterial infections, etc.; or (III) preoperative determination by the anesthesiologist that endotracheal intubation and general anesthesia could be tolerated; (IV) Eastern Cooperative Oncology Group performance status (ECOG PS) score $>2$.

\section{Surgical procedures}

\section{NSS group}

The procedure conducted in the NSS group has been described previously (8). Patients were placed in a supine position with their head back. Anesthesia included singlelumen endotracheal intubation. A 4-cm incision was made along the anterior border of the sternocleidomastoid muscle (Figure $1 A, 1 B$ ), and then standard dissection of the cervical esophagus was conducted. A wound protector was placed and covered with a glove to seal the incision. $\mathrm{CO}_{2}$ was insufflated into the mediastinum up to a pressure of 8 to $10 \mathrm{mmHg}$. The esophagus was dissected up to the level of the lower pulmonary vein. Dissection of the bilateral recurrent laryngeal nerve (RLN), paraesophageal lymph nodes and subcarinal lymph nodes was performed, and a small incision was added in the right neck to remove the right paralaryngeal nerve lymph nodes when necessary. Standard laparoscopy was then used to dissect the stomach. The lower thoracic esophagus was separated through the esophageal hiatus, and the lymph nodes on the side of the cardiac and lower pulmonary veins were cleared. After the above operation, a vertical incision of $5 \mathrm{~cm}$ was made under the xiphoid process, and the stomach was pulled out from the body to form a tubular stomach (10). The tubular stomach was pulled to the neck through the posterior mediastinum. Finally, the anastomosis was performed with a tubular stapler.

\section{SS group}

First, the patient was placed in a supine position, and then general anesthesia was induced. The second step involved making a 4-cm neck incision along the medial side of the left sternocleidomastoid at the jugular notch on the superior margin of the manubrium sternum (Figure 1A,1B). Next, the fixation rod of the sternum suspension retractor (The sternum suspension retractor is a medical device. The manufacturer: Suzhou Wino Yikang Medical Equipment Co., Ltd., Model: SCL01) was installed on the left side of the operating bed (Figure 1C,1D). Following this, a $0.5 \mathrm{~cm}$ incision was made in the third or fourth intercostal space of the left margin of the sternum (Figure $1 A, 1 B$ ). After a small amount of separation was created, the sternum suspension retractor hook was inserted behind the sternum (Figures $1 B, 1 D, 2 A, 2 B)$. Then, the retractor hook that had been inserted behind the sternum to the fixation rod was connected (Figures $1 C, 1 D, 2 A, 2 B$ ). At this point, the sternum could be raised 5 to $10 \mathrm{~cm}$ by adjusting the suspension 

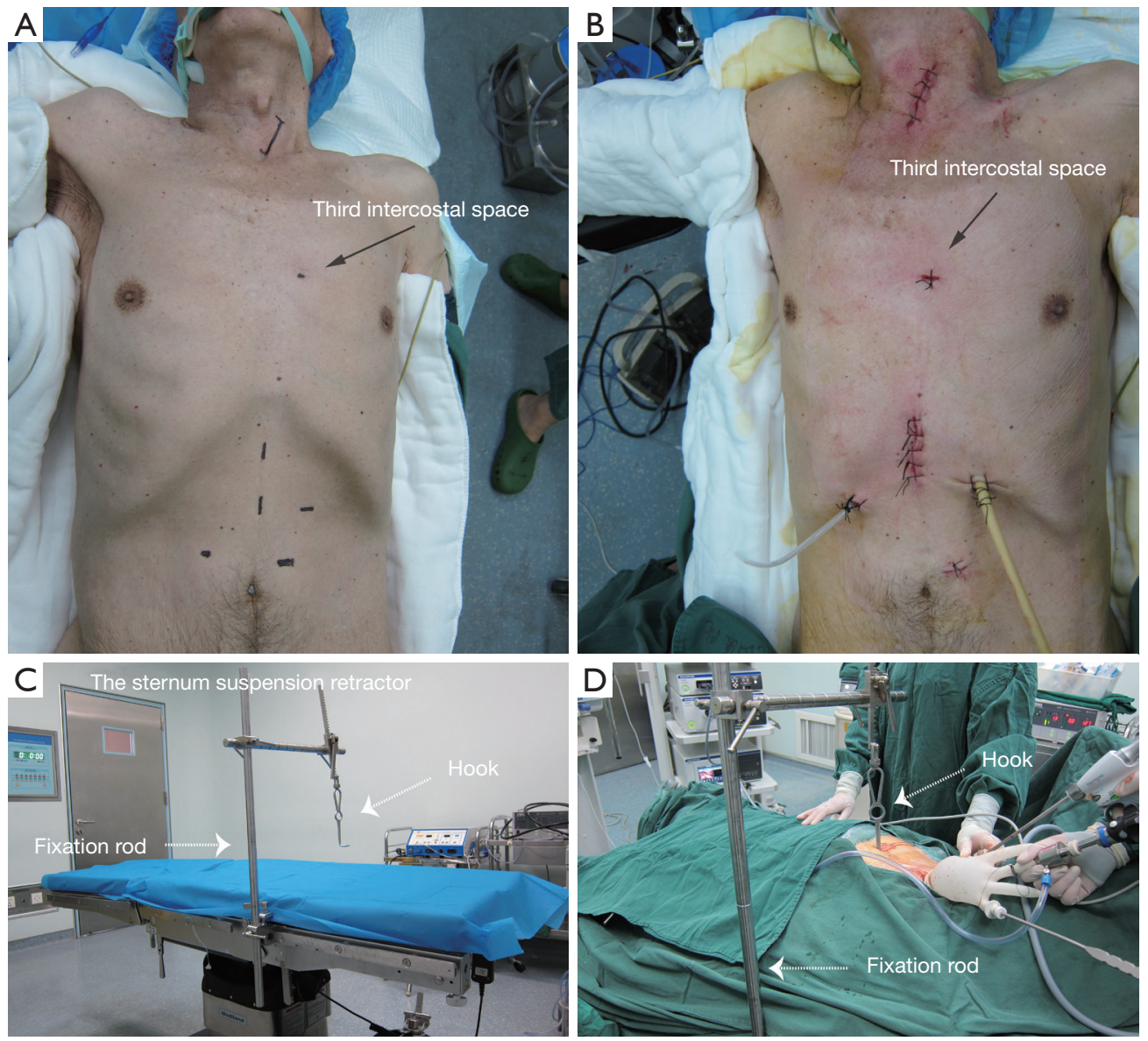

Figure 1 Surgical incision and the sternal suspension retractor. (A) Schematic diagram of incision preoperatively. (B) Postoperative incision. (C) Sternum suspension retractor. (D) Lifting the sternum.

retractor (Figure 2B). When the lower esophagus is separated by laparoscope, the left atrium can be compressed by the abdominal retractor (Figure 2C). Moreover, the trocar with abdominal retractor on the abdominal wall has no fixed fulcrum and is easy to move. So we used sterile ropes to attach the trocar to the fixation rod and the retractor hook, which are used for sternal suspension (Figure 2D). The rest of the procedure was the same as that performed in the NSS group $(8,10)$.

\section{Postoperative care}

All patients were treated with anti-infective prophylactic therapy, with intravenous or enteral nutrition being administered concurrently, and a full or half fluid diet was returned on days 5 to 7 after surgery. Routine treatment of analgesia, atomization, and expectorant was given after the operation. Hoarseness was assessed on the first postoperative day.

\section{Data collection}

The clinical data of all patients were collected. Postoperative follow-up was conducted for 1 month and once every 3 months by telephone and outpatient clinic appointment. Intraoperative and postoperative clinical data including post-surgical pathologic TNM classification (pTNM), post-surgical pathologic tumor classification (pT), postsurgical pathologic extent of lymph node involvement $(\mathrm{pN})$, intramediastinal operation time, intraoperative blood loss, number of thoracic lymph nodes, total number of dissected lymph nodes, postoperative complications (hoarseness, anastomotic fistula, pulmonary infection, arrhythmia, chylothorax), and postoperative hospital stay were evaluated. 

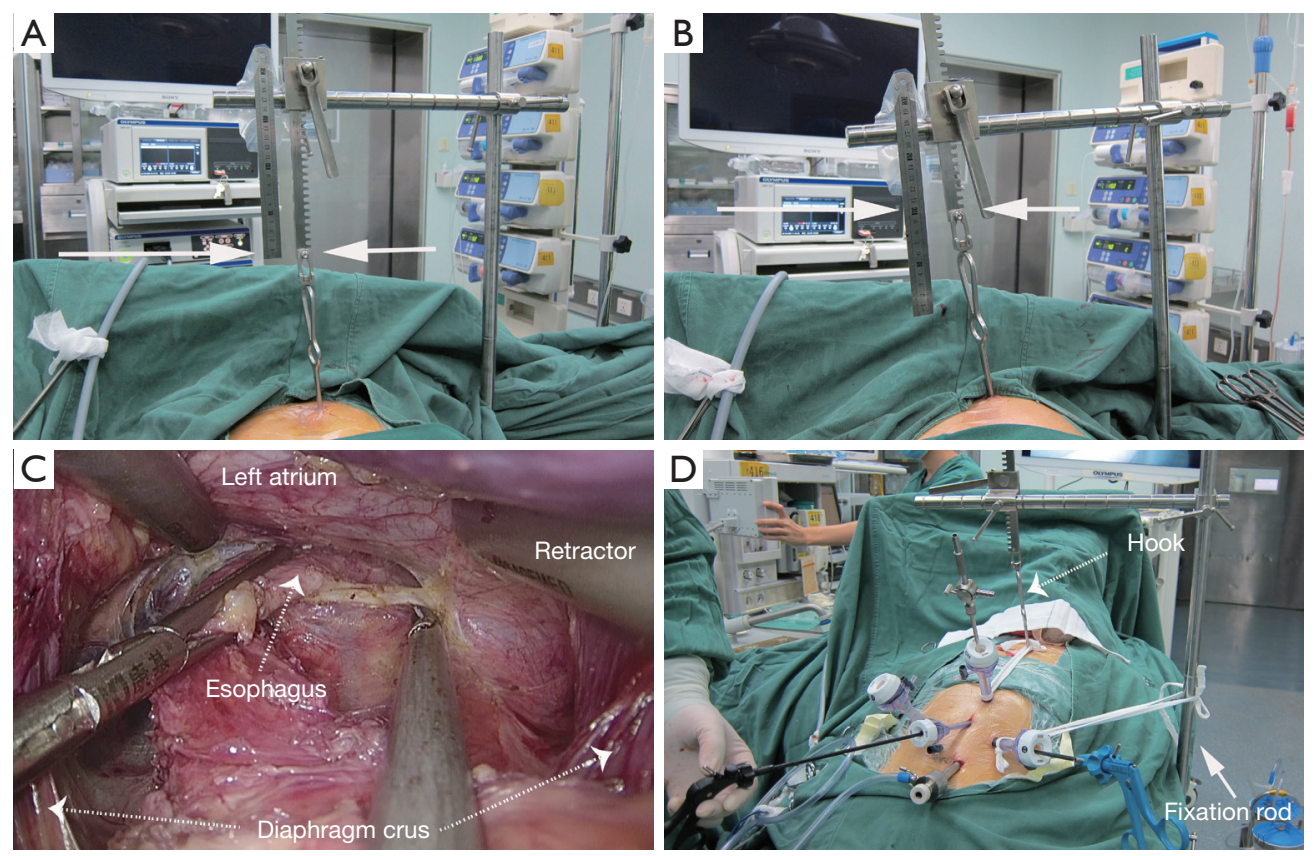

Figure 2 Sternal suspension retractor in action. (A) Before the sternum is raised. (B) The sternum is raised about $10 \mathrm{~cm}$ (white arrows). (C) Lifting sternum reduces compression of the mediastinal retraction on the left atrium. (D) The trocar location of the abdomen when the sternum is elevated.

We chose the number of thoracic lymph nodes instead of the total number of dissected lymph nodes for comparison in order to avoid confounders.

\section{Statistical analysis}

IBM SPSS Statistics for Windows, version 20 software (IBM Corp., Armonk, NY, USA) was used to collect and analyze the clinical data of all patients. Continuous variables are represented by mean \pm standard deviation, and the $t$-test was used for comparison. When the data were nonnormally distributed variables, the median and quartile spacing are used to represent the central trend and the degree of dispersion, and the rank-sum test was used for testing. The categorical variables were compared by the $\chi^{2}$ test or Fisher's exact test. A $\mathrm{P}$ value $<0.05$ was considered significant.

\section{Results}

All patients in the two groups were successfully operated on according to the preoperative plan, and there was no transfer to open surgery. All patients in both groups received R0 resection. There were no serious complications, such as intraoperative hemorrhage, trachea injury, or thoracic duct injury in the two groups.

During these operations, we found that sternal lifting improved visibility by increasing the working space and working angles available through the mediastinoscopy ports. The elevated upper margin of the manubrium sternum raised the corresponding side's cervical incisional protective sleeve. Furthermore, this also adjusted the incisional protective sleeve to a slightly upright position (Figure $3 A, 3 B$ ). This angle was more convenient for the intraoperative surgical instruments to pass through the incisional protective sleeve into the mediastinal operating field (Figure 3B). Most importantly, it successfully enlarged the intramediastinal operative space (Figure $3 C, 3 D$ ). The distance between the trachea and the thoracic vertebrae in the operative field was significantly larger after the sternal suspension (Figure 3D), and thus it was significantly easier to dissociate the esophagus. We also found this helpful for identifying the azygos arch, thoracic duct, trachea, carina, and bronchus, in avoiding damage to these tissues. Our suspension widened the mediastinal space when the esophagus was laparoscopically dissociated from the lower mediastinum through the diaphragm hiatus, thus reducing 
A
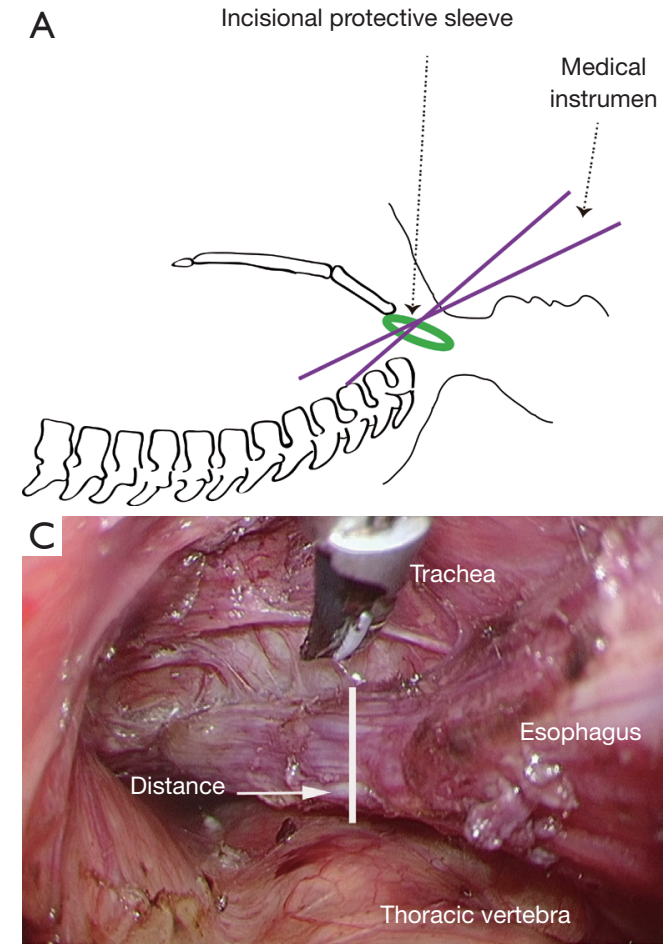

B Incisional protective sleeve
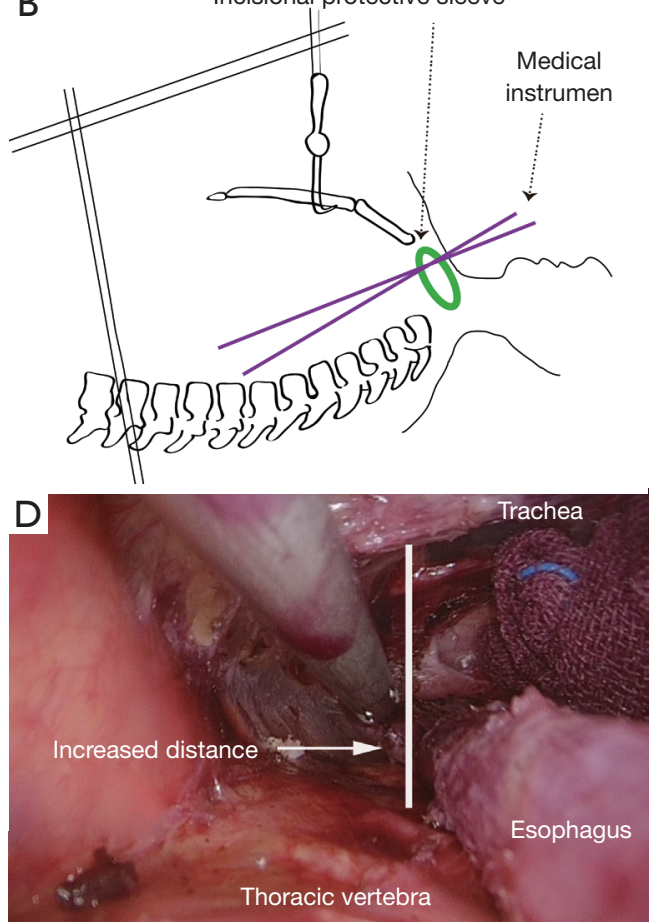

Figure 3 The contrast of before and after the sternum is elevated. (A) Schematic diagram for the inclination of the neck incisional protective sleeve without lifting the sternum. (B) A schematic diagram of how lifting the sternum can lead to a steep angle of the neck incisional protective sleeve. (C) Intramediastinal space without lifting sternum. The distance from the trachea to the thoracic vertebra is shorter when the sternum is not raised. (D) Lifting the sternum enlarges the intramediastinal operative space. The distance from the trachea to the thoracic vertebra has increased.

the compression of the mediastinal retractor on the left atrium (Figure 2C,2D).

In the comparison of the two groups' data, intramediastinal operation time was significantly shorter in the SS group than in the NSS group (82.50 vs. 110.00 minutes; $\mathrm{P}<0.001)$. For chest lymph nodes, the SS group showed more dissected lymph nodes than did the NSS group 14 vs. $12 ; \mathrm{P}=0.036$ ). Patients in the SS group had a lower incidence of postoperative hoarseness than did those in the NSS group $(2 / 20$ vs. $6 / 19 ; \mathrm{P}=0.235)$. There were no significant differences between the SS group and NSS group in terms of intraoperative blood loss, postoperative hospital stay, pTNM stages, $\mathrm{pT}, \mathrm{pN}$, or the total number of dissected lymph nodes. Additionally, there were no statistical differences in the incidence of anastomotic fistula, respiratory complications, arrhythmia, or chylothorax between the two groups (Table 2), nor was there any mortality during hospitalization in the two groups.

In the SS group, none of the patients who underwent sternal suspension had an intercostal nerve, vascular, cardiac, or internal thoracic vascular injury during or after surgery. There was no bleeding, infection, or reported pain from the intercostal incision for suspension of the sternum.

All patients were followed up by telephone and outpatient follow-up, with a median follow-up time of 16.3 months (range, 3 weeks to 29 months). During routine follow-up, 1 patient in the SS group developed liver metastasis. Lung metastasis occurred in 1 case in the NSS group.

\section{Discussion}

Combined single-port mediastinoscopic and laparoscopic access with $\mathrm{CO}_{2}$ insufflation in esophagectomy has gradually become an area of intense research interest since first being reported by Fujiwara et al. in $2015(3,9)$. Compared with transthoracic esophagectomy, VARETT ensures a relatively intact chest with less interference to the heart and lungs. After surgery, there was no concern for chest incision pain. 
Table 2 Comparison of clinical data between the two groups

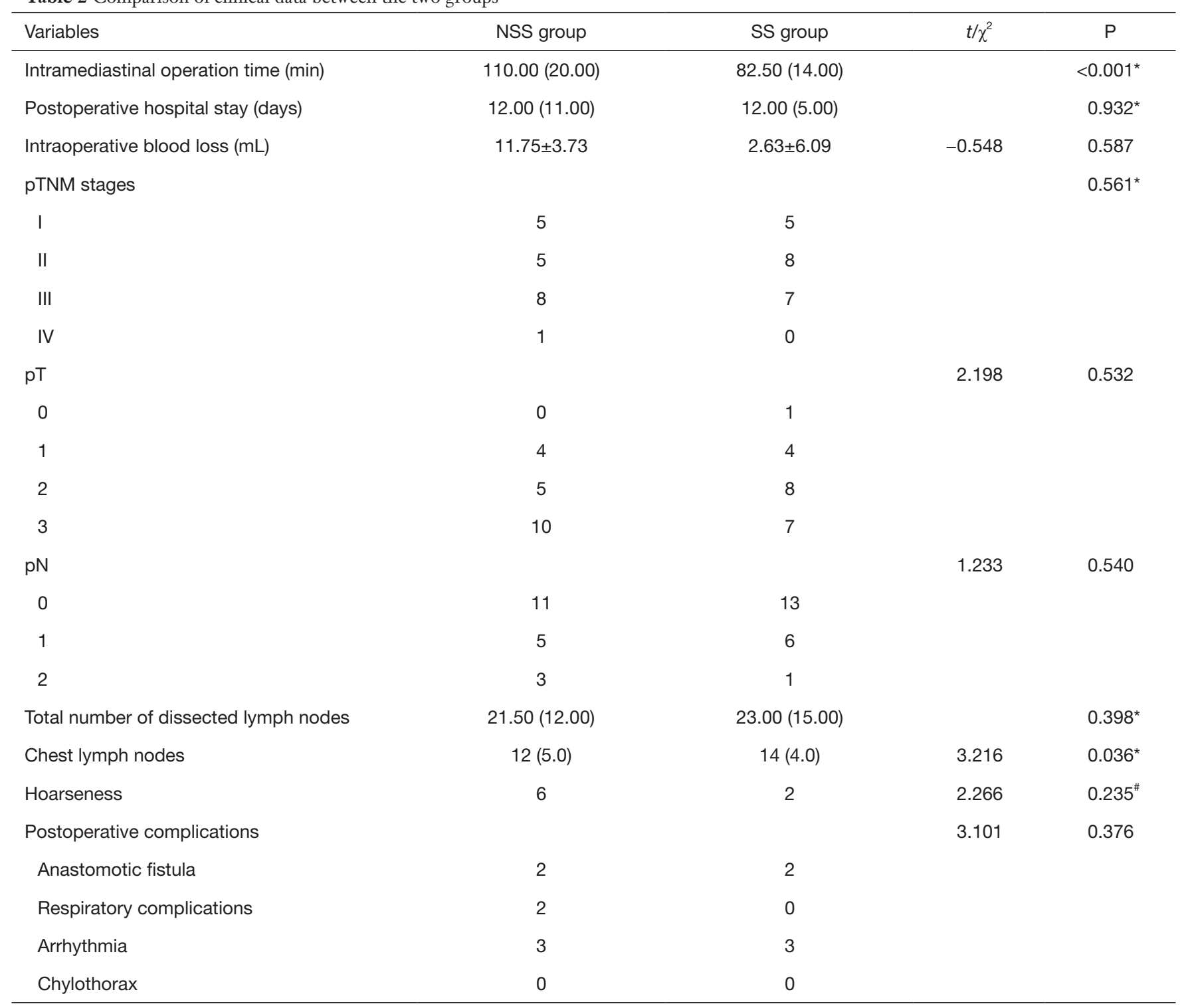

*, indicates variables that are not normally distributed. Chest operation time, postoperative hospital stay, and total number of lymph nodes had a nonnormal distribution; median and quartile spacing are used to represent the central trend and degree of dispersion. The $\mathrm{P}$ value obtained by the rank-sum test. ", the P value of hoarseness was obtained using Fisher's exact probability method. NSS group, nonsternal suspension group; SS group, sternal suspension group; pTNM, pathologic TNM classification; pT, post-surgical pathologic tumor classification; pN, post-surgical pathologic extent of lymph node involvement.

Several comparative studies have confirmed the feasibility of VARETT, with similar therapeutic outcomes to those of transthoracic esophagectomy during long-term follow-up $(6,11)$. VARETT avoids transthoracic surgery and is better suited for patients with poor heart and lung function or those with a history of pleural disease. It does not require changing the patient's position during surgery, while the duration of the entire operation is relatively short.

However, due to the narrow operating space of the free esophagus under mediastinoscopy, VARETT indications and contraindications are still controversial, and there is no accepted standard. Because important tissues and organs, such as the aorta, trachea, heart, azygos vein arch, bronchial artery, and thoracic duct are located near the esophagus, 
it is easy to damage these important adjacent structures during the operation in the narrow mediastinal space (10). In addition to this, systematic lymph node dissection is very important in the radical operation of esophageal cancer. Some scholars have raised concerns regarding the dissection of right RLN lymph nodes and subcarinal lymph nodes, as this may lead to the operation not conforming to the principles of tumor surgical treatment. Thus, it has a strict indication; that is, it is only suitable for $\mathrm{T} 1$ or $\mathrm{T} 2$ patients, in whom there is no invasive mass and no metastasis in the mediastinal lymph node. Consequently, VARETT has not been widely applied $(3,9)$. A few researchers have reported on various improvement to the instruments and methods involved in this approach, but standard techniques have not yet been established (12).

We first applied sternal suspension retractors to VARETT. Use of the sternal suspension retractor has been previously reported for total thymectomy, which can enlarge the space during operation $(13,14)$. After the sternum was suspended in VARETT, the intramediastinal operative field was enlarged, which was conducive to esophageal dissociation, particularly when the tumor exceeded the outer membrane of the esophagus. In particular, the increased visual field helped to avoid damage to the blood vessels, thoracic duct, and tracheal and bronchial membrane. Once a small hemorrhage occurs, the expanded field of view makes it easy to stop the bleeding under the mediastinoscope. In addition, the raised sternum by the suspension hook reduces the inclination of the neck incision protective sleeve above the sternum handle, facilitating the entry of the instrument into the mediastinum, and increasing the ease of operation. Due to the above advantages, in our study, compared with the NSS group, the SS group experienced a significantly shortened time in freeing the esophagus in the mediastinum, had more cleared chest lymph nodes, was less likely undergo damage to the RLN, and had a lower incidence of postoperative hoarseness. Some scholars have confirmed that lymph node dissection in VARETT can meet the requirements of radical esophagectomy $(3,15)$. In VARETT, our team focused on the dissection of bilateral RLN lymph nodes and subcarinal lymph nodes and added a small incision in the right neck to remove the right paralaryngeal nerve lymph nodes when necessary. The low incidence of hoarse voice in the SS group suggests that sternal suspension can increase the operation space of the mediastinum, thus reducing the incidence of vocal cord paralysis. However, due to the small sample size of patients in this operation, there may be bias, so a large sample of experiments is needed to verify this assumption.

In our study, no negative intraoperative injuries, such as intercostal nerves, blood vessels, heart, or intrathoracic vessels were observed in patients who received sternal suspensions. During postoperative observation, there were no reports of bleeding, infection, or pain from the intercostal incision of the sternal suspension. These findings suggest that the addition of sternal suspension in VARETT is safe and feasible.

We believe that the addition of sternal suspension in VARETT can safely complete the operation to the thoracic esophagus, and the advantages of sternal suspension can expand the operation space of the mediastinum. We believe that with the proficiency of the VARETT technique, sternal suspension may expand the clinical application of VARETT and thus have great application value (16). As this is the first time that sternal suspension was combined with VARETT, a small number of patients were included in this study, making a degree of selection bias inevitable. Additional sample sizes are needed to confirm the advantages of sternal suspension.

\section{Conclusions}

Based on the results of this study, we believe that sternal lifting increases the operating space in esophagectomy via mediastinoscopy, that sternal suspension can make VARETT easier to perform, and that sternal suspension in VARETT is safe and effective. We expect the further development and refinement of this technique will expand the indications for VARETT.

\section{Acknowledgments}

Funding: This study was supported by the Nature and Science Fund of Key Cultivate Program from Wannan Medical College, CN (grant No. WK2018ZF06), the Science Research Fund for Talent Introduction of Yijishan Hospital of Wannan Medical College (grant No. YR201806), and the Science Fund for Key Program of Three Creative Research of Yijishan Hospital of Wannan Medical College (grant No. Z1810).

\section{Footnote}

Reporting Checklist: The authors have completed the STROBE reporting checklist. Available at https://dx.doi. org/10.21037/jtd-21-1406 
Data Sharing Statement: Available at https://dx.doi. org/10.21037/jtd-21-1406

Conflicts of Interest: All authors have completed the ICMJE uniform disclosure form (available at https://dx.doi. org/10.21037/jtd-21-1406). Dr. SZ reports funding from the Nature and Science Fund of Key Cultivate Program from Wannan Medical College, CN (grant No. WK2018ZF06); the Science Research Fund for Talent Introduction of Yijishan Hospital of Wannan Medical College (grant No. YR201806) and the Science Fund for Key Program of Three Creative Research of Yijishan Hospital of Wannan Medical College (grant No. Z1810). The other authors have no conflicts of interest to declare.

Ethical Statement: The authors are accountable for all aspects of the work in ensuring that questions related to the accuracy or integrity of any part of the work are appropriately investigated and resolved. All patients undergoing surgery signed informed consent forms. The design of the study was carried out in strict accordance with the principles of the Declaration of Helsinki (2013 revision). The study was approved by the institutional ethics committee of Yijishan Hospital of Wannan Medical College (No.: 73).

Open Access Statement: This is an Open Access article distributed in accordance with the Creative Commons Attribution-NonCommercial-NoDerivs 4.0 International License (CC BY-NC-ND 4.0), which permits the noncommercial replication and distribution of the article with the strict proviso that no changes or edits are made and the original work is properly cited (including links to both the formal publication through the relevant DOI and the license). See: https://creativecommons.org/licenses/by-nc-nd/4.0/.

\section{References}

1. Ahmed M, Lau A, Hirpara DH, Kidane B. Choosing the right survey—patient reported outcomes in esophageal surgery. J Thorac Dis 2020;12:6902-12.

2. Wang J, Hu J, Zhu D, Wang K, et al. McKeown or Ivor Lewis minimally invasive esophagectomy: a systematic review and meta-analysis. Transl Cancer Res 2020;9:1518-27.

3. Fujiwara H, Shiozaki A, Konishi H, et al. Singleport mediastinoscopic lymphadenectomy along the left recurrent laryngeal nerve. Ann Thorac Surg 2015;100:1115-7.

4. Wang X, Li X, Cheng H, et al. Single-port inflatable mediastinoscopy combined with laparoscopic-assisted small incision surgery for radical esophagectomy is an effective and safe treatment for esophageal cancer. J Gastrointest Surg 2019;23:1533-40.

5. Jin $\mathrm{Y}, \mathrm{Lu} X, \mathrm{Xue} \mathrm{L}$, et al. Retrospective comparison of two minimally invasive esophagectomy in the treatment of esophageal cancer: pneumatic mediastinoscopy versus thoracoscopy. J Laparoendosc Adv Surg Tech A 2019;29:638-42.

6. Koide N, Takeuchi D, Suzuki A, et al. Mediastinoscopyassisted esophagectomy for esophageal cancer in patients with serious comorbidities. Surg Today 2012;42:127-34.

7. Feng MX, Wang H, Zhang Y, et al. Minimally invasive esophagectomy for esophageal squamous cell carcinoma: a case-control study of thoracoscope versus mediastinoscope assistance. Surg Endosc 2012;26:1573-8.

8. Zhu S, Yu S, Liu F. Combined single-port transmediastinal and laparoscopic access with $\mathrm{CO} 2$ insufflation for esophageal resection: a case report on a canine model. AME Case Rep 2020;4:6.

9. Fujiwara H, Shiozaki A, Konishi H, et al. Mediastinoscope and laparoscope-assisted esophagectomy. J Vis Surg 2016;2:125.

10. Liu W, Guo X, Zhao H, et al. Mediastinoscopy-assisted transhiatal esophagectomy versus thoraco-laparoscopic esophagectomy for esophageal cancer: a single-center initial experience. J Thorac Dis 2020;12:4908-14.

11. Wang QY, Tan LJ, Feng MX, et al. Video-assisted mediastinoscopic resection compared with video-assisted thoracoscopic surgery in patients with esophageal cancer. J Thorac Dis 2014;6:663-7.

12. Wu CL, Dong B, Wu B, et al. The application of rigid and flexible mediastinoscopy in esophagectomy: our experience and a new technology. World J Surg Oncol 2021;19:234.

13. Aubert A, Chaffanjon P, Brichon PY. Video-assisted extended thymectomy in patients with thymoma by lifting the sternum: is it safe? Ann Thorac Surg 2004;77:1878; author reply 1878 .

14. Takeo S, Sakada T, Yano T. Video-assisted extended thymectomy in patients with thymoma by lifting the sternum. Ann Thorac Surg 2001;71:1721-3.

15. Fujiwara H, Shiozaki A, Konishi H, et al. Hand-assisted laparoscopic transhiatal esophagectomy with a systematic procedure for en bloc infracarinal lymph node dissection. 
Dis Esophagus 2016;29:131-8.

16. Okumura T, Seto Y, Aikou S, et al. Mediastinoscopic salvage esophagectomy for recurrent esophageal squamous cell carcinoma after definitive chemoradiotherapy in a previously pneumonectomized patient. Asian J Endosc Surg 2019;12:452-6.

(English Language Editor: J. Gray)

Cite this article as: Zhu S, Zhang G, You Q, Li F, Ding B, Liu F, Ma T. Sternal lifting increases the operating space in esophagectomy via mediastinoscopy: a prospective cohort study. J Thorac Dis 2021;13(9):5546-5555. doi: 10.21037/jtd-21-1406 